\title{
Reviewer's comment to Letter to the Editor of Jingming Lin et al. concerning "Comparison of unilateral versus bilateral pedicle screw fixation in lumbar interbody fusion: a meta-analysis" (by W. Ding et al. (2014) Eur Spine J 23(2):395-403): and authors' reply of Wenbin Ding et al.
}

\author{
Kimberley Edwards ${ }^{1}$
}

Received: 3 July 2015/Revised: 3 July 2015/Accepted: 3 July 2015 / Published online: 24 July 2015

(c) Springer-Verlag Berlin Heidelberg 2015

1. The letter authors comment that the review "did not focus specifically or in any details on the issue of the completeness of the search strategy report for databases". I completely agree.

The original authors have addressed this point to some degree in their response to the letter, but it is still weak. For example, in the original article it says, for example: "randomized controlled trial (RCT) is preferred [to prospective study]" - how was this evaluated/did it make a difference to inclusion or not? I note in figure 1 it only refers to trials; Similarly, the comment that "patients were diagnosed with lumbar degenerative diseases according to symptoms, signs and imaging data" is far too vague.

2. The letter authors also say "the investigators did not follow the Preferred Reporting Items for Systematic Reviews and Meta-Analyses (PRISMA)". Again I completely agree-the authors should have followed PRISMA and should have stated this in their paper.

The authors say (in their response to this letter) that they have followed the PRISMA guidance (http://www.prismastatement.org/), but Figure 1 shows that this is not entirely correct. Authors should report four stages-i.e. 'identification', 'eligibility', 'screening' and 'included'. Ding et al. have combined the middle two stages into one.

3. The letter authors ask that "the studies should be combined by DerSimonian and Laird random-effects model [for the three secondary outcomes]". Here, I do not agree with the letter. The review authors did use a random effects model for these three secondary outcomes, which is reported in the paper (and they reiterate this in their letter).

4. Similarly, the letter states that "the investigators did not mention the heterogeneities. We suggest that the investigators should analysis the reasons for the significant heterogeneities". I disagree. The original paper does clearly state the $\mathrm{I}^{2}$ values (in the text and figures). What the original authors do not do is suggest reasons for the heterogeneities, which they have addressed to some degree in their response to the letter.

Compliance with ethical standards

Conflict of interest None.
Kimberley Edwards

Kimberley.Edwards@nottingham.ac.uk

1 Academic Orthopedics, Trauma and Sports Medicine, University of Nottingham, Floor C, West Block, Queen's Medical Centre, Nottingham NG7 2UH, UK 